\title{
Second-hand smoke exposure higher for kids and teens
}

$\mathrm{E}$ xposure to second-hand smoke is generally declining slightly in Canada, according to recently released biomonitoring data, but levels of a biomarker for tobacco smoke are still higher in children and teens than in adults.

"It's making progress, but we're not declaring 'mission accomplished' just yet," said Dr. Atul Kapur, president of Physicians for a Smoke-Free Canada and an emergency physician in Ottawa.

The data are from the third "cycle" of human biomonitoring of environmental chemicals in Canada, released July 15 . Blood and urine samples to screen for 48 chemicals were collected as part of the Canadian Health Measures Survey conducted by Statistics Canada, in collaboration with Health Canada and the Public Health Agency of Canada, between January 2012 and December 2013. Samples are from about 5800 Canadians aged 3 to 79 years at 16 sites across Canada. The first cycle was published in 2010 based on data collected 2007-2009, and the second cycle was published in 2013 based on 2009-2011 data.

Chemicals screened for include cotinine, a metabolite of nicotine, which indicates exposure to tobacco smoke. Kapur said, "cotinine level is not $100 \%$ matched with second-hand smoke, but it's pretty good," and is often used as a biomarker. "It's got a half-life of 16 to 20 hours, so it will hang around for a few days."

The percentage of those tested with detectable levels is the important number, said Kapur. Each cycle of biomonitoring has shown a lower level, both overall and in various age groups. "In all ages, we've gone from $13 \%$ to less than 12\%" between the 2009-2011 cycle and the 2012-2013 cycle, "so it's moved a little bit."

However, Kapur is concerned about levels in children and teens. "The level of kids who have detectable cotinine is

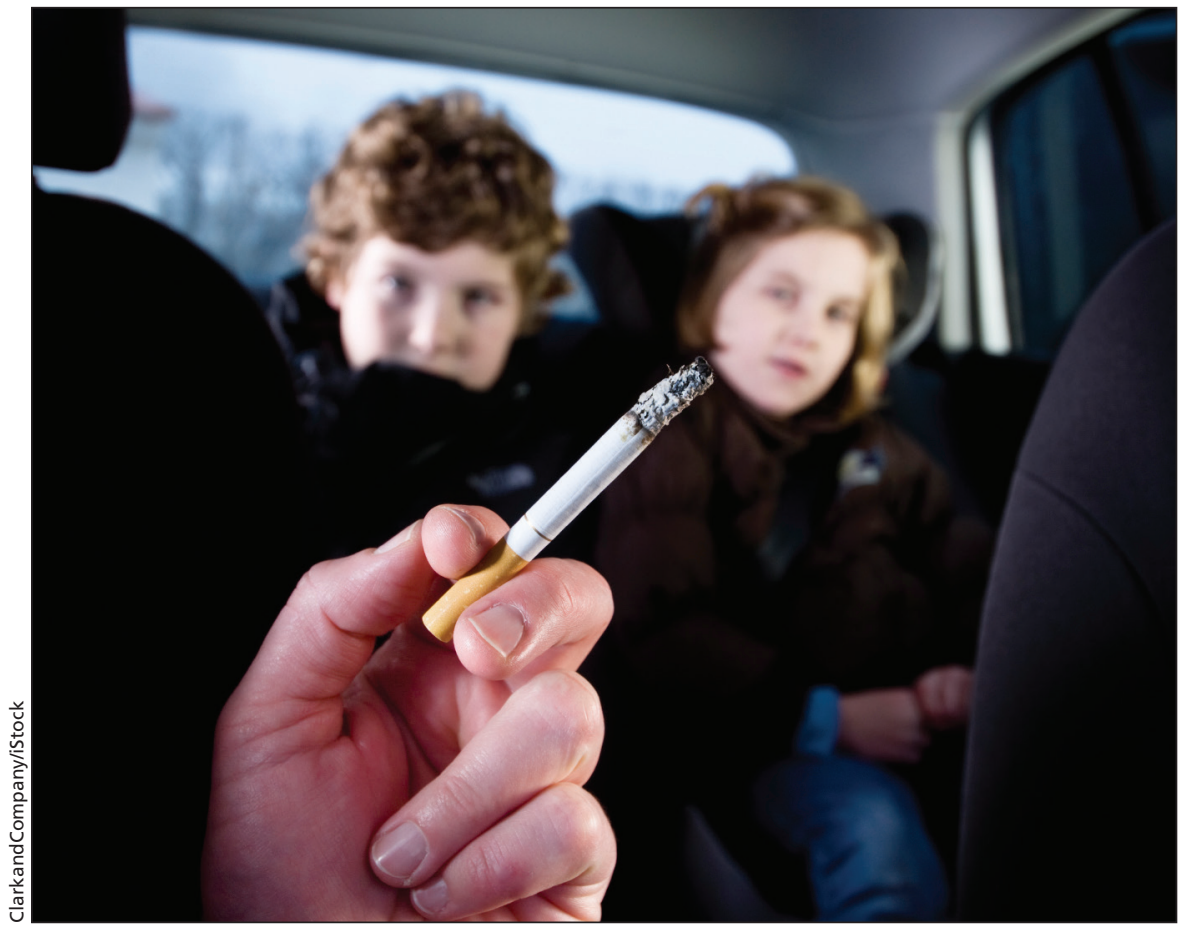

Children may have higher exposure to second-hand smoke because they are less able to influence adult smoking behaviour.

higher than for adults," a finding that has been consistent in every cycle of the biomonitoring data. In the most recent data, almost $12 \%$ of preschoolers have detectable levels, as do $13 \%$ of those age 6 to 11, and almost $18 \%$ of those age 12 to 19 . "Those numbers show that's there's still significant numbers of kids who are exposed to second-hand smoke."

By contrast, the percentage of people age 20 to 40 with detectable levels has declined from almost $14 \%$ in cycle 2 to less than $10 \%$ in cycle 3 .

The biomonitoring data are in line with results from the Canadian Community Health Survey (CCHS), in which $12.6 \%$ of 12 - to 19 -year-olds surveyed in 2014 said they were exposed to smoke in the home (younger children were not surveyed). "Children are less able to influence what the situation is in the home, whereas adults have more ability to avoid smoke," Kapur believes.
Exposure in public places is still common for people of all ages, the CCHS shows. In 2008, 9.9\% of nonsmokers reported exposure; in 2014, that increased to $13.5 \%$.

Among people who smoke tobacco, the biomonitoring data show cotinine levels have also declined slightly, although cotinine levels in smokers remain hundreds of times higher than in nonsmokers.

"The confidence intervals are very wide, so this may not be a real change," says Kapur. "Hopefully it would indicate that among smokers that they are smoking less, but I can't say that with real certainty."

Lower exposure to second-hand smoke may also lower cotinine levels in smokers. "It may very well be a virtuous circle," said Kapur. - Carolyn Brown, Ottawa, Ont.

CMAJ 2015. DOI:10.1503/cmaj.109-5148 